\title{
Molecular epidemiology of invasive methicillin-susceptible Staphylococcus aureus strains circulating at a Swiss University Hospital
}

\author{
L. Fenner • A. F. Widmer • R. Frei
}

Received: 31 July 2007 / Accepted: 14 January 2008 / Published online: 26 February 2008

(C) Springer-Verlag 2008

\begin{abstract}
In contrast to methicillin-resistant Staphylococcus aureus, little is known of the distribution of spa types among methicillin-susceptible $S$. aureus (MSSA). We have analyzed 101 nonrepetitive invasive MSSA isolates from infected patients, consecutively isolated during 14 months between 2006 and 2007 at University Hospital Basel. They were genetically characterized according to $S$. aureus protein A $(s p a)$ types and important virulence-associated genes. Sixty-five different spa types corresponding to nine different spa clonal complexes were observed. Analysis of different virulence genes showed a frequency of $17 \%$ for toxic-shock syndrome toxin and 5\% for exfoliative toxin D. In conclusion, spa typing revealed a great genetic diversity without predominant spa type, not providing evidence for clonal spreading.
\end{abstract}

\section{Report}

Staphylococcus aureus is an important human pathogen and is isolated in up to $25 \%$ of nosocomial infections. S. aureus bacteraemia is associated with substantial excess morbidity and mortality worldwide [1]. A recent study from the

L. Fenner $\cdot$ R. Frei $(\bowtie)$

Microbiology Laboratory, University Hospital Basel,

Petersgraben 4,

4031 Basel, Switzerland

e-mail: rfrei@uhbs.ch

\section{A. F. Widmer}

Division of Infectious Diseases and Hospital Epidemiology, University Hospital Basel,

Basel, Switzerland
United States showed a predominance of one clonal complex (ST8) among methicillin-susceptible S. aureus (MSSA) strains, whereas the remaining strains were genotypically heterogeneous [2]. Strain typing is an established tool for surveillance and determination of strain relatedness. Sequence-based methods such as protein A gene $(s p a)$ strain typing offer the opportunity of reproducible and comparable results and have been successfully used in epidemiological studies [3, 4]. To date, relatively little data exist on spa types and the molecular epidemiology of invasive MSSA strains in contrast to methicillinresistant $S$. aureus (MRSA). In this study, our goal was to assess the frequency of circulating invasive MSSA spa types isolated from primarily sterile sites in patients at University Hospital Basel (UHBS), Switzerland, and to detect virulence associated genes such as Panton-Valentine leukocidin (PVL), toxic shock syndrome toxin (tst), exfoliative toxin A, B, and D (eta, etb, etd).

As standard operating procedure, invasive MSSA isolates from primarily sterile sites (cerebro-spinal fluid, sterile body fluids, biopsies, blood cultures) are routinely saved at $-70^{\circ} \mathrm{C}$ at our laboratory. The isolates were identified as $S$. aureus on the basis of positive catalase, clumping factor (Slidex StaphPlus, bioMérieux, Marcy l'Etoile, France), and aurease (RAPIDECstaph, bioMérieux). Only one isolate per patient was included. DNA was extracted with LC MagnaPure system (Roche Diagnostics, Rotkreuz, Switzerland) according to manufacturer's instruction. The spa gene was amplified using primers spa-1113f and spa-1514r and sequencing was performed as previously described [4]. Sequences were analyzed with Ridom StaphType software version 1.4 (Ridom GmbH, Würzburg, Germany). Clustering analysis into spa clonal complexes (spa-CC) was done 
using default parameters (spa types are clustered if cost is less than or equal to 6 and spa types shorter than 5 repeats are excluded). PVL, tst, eta, etb, and etd genes were detected by PCR as described elsewhere [5-7]. Amplification of the 16S rRNA gene was used to confirm absence of PCR inhibitors. PCR products were visualized by electrophoresis on ethidium bromide-stained 3\% agarose gels. Antibiotic susceptibility was tested applying a microbroth dilution procedure (MERLIN Diagnostika, Bornheim-Hersel, Germany) and interpreted in accordance with the Clinical and Laboratory Standards Institute (http://www. clsi.org).

A total of 105 patients with MSSA infection during a 14month period in 2006-2007 were recorded. Isolates of 101 patients were analyzed; four isolates were not available.
The median age of patients was 61 years (interquartile range 45-74) with $63.4 \%$ being males. The highest proportions of MSSA strains were isolated from the surgical department (42\%), emergency department (31\%), internal medicine $(11 \%)$, and intensive care units $(10 \%)$. Blood culture was the most common body site; the same organism was isolated from another source in $11.2 \%$ (9 isolates; not included to avoid double counting). Specimens from joint, bone, and skin/soft tissue each accounted for $5.9 \%$ (6 isolates). A total of 65 different spa types corresponding to nine different spa-CC were observed (Table 1), and 51 strains had a unique spa type. Eleven newly recognized spa types were found which have never been deposited before in the spa database (http:// www.spaserver.ridom.de). The ten most frequent spa types

Table 1 Distribution of 101 invasive methicillin-susceptible S. aureus (MSSA) strains by spa clonal complex (spa-CC), presence of virulenceassociated determinants, and antibiotic resistance profile

\begin{tabular}{|c|c|c|c|c|c|c|c|c|c|c|c|}
\hline \multirow[t]{2}{*}{ Spa-CC } & \multirow[t]{2}{*}{ Spa Type } & \multirow[t]{2}{*}{ Frequency } & \multirow[t]{2}{*}{ PVL } & \multirow[t]{2}{*}{ etd } & \multirow[t]{2}{*}{ tst } & \multirow[t]{2}{*}{ eta/etb } & \multirow[t]{2}{*}{ Penicillin R } & \multirow[t]{2}{*}{ Clarithromycin $\mathrm{R}$} & \multirow[t]{2}{*}{ Quinolone $\mathrm{R}^{\mathrm{b}}$} & \multicolumn{2}{|c|}{ Site of Isolation } \\
\hline & & & & & & & & & & Blood & $\begin{array}{l}\text { Other than } \\
\text { blood }\end{array}$ \\
\hline & & $n$ & $n$ & $n$ & $n$ & $n$ & $n(\%)$ & $n(\%)$ & $n(\%)$ & $n$ & $n$ \\
\hline $\mathrm{CC} 084 / 085$ & $\begin{array}{l}\text { t084 (8), t085 (1), t091 (1), } \\
\text { t094 (1), t254 (1), t279 (1), } \\
\text { t346 (2), t803(1), t2432(1), } \\
\text { t2434 (1) }\end{array}$ & 18 & & & & 1 & $16(89)$ & & & 15 & 3 \\
\hline $\mathrm{CC} 012$ & $\begin{array}{l}\text { t012 (3), t021 (2), t093 (1), } \\
\text { t122 (2), t298 (1), t665 (1), } \\
\text { t913 (1), t1306 (1), t1836(1), } \\
\text { t2437 (1) }\end{array}$ & 14 & 1 & & 8 & & $13(93)$ & & $1(7.1)$ & 11 & 3 \\
\hline $\mathrm{CC} 002$ & t002 (7), t062 (2), t548 (2) & 11 & & & 5 & & $5(45.5)$ & $3(27.3)$ & $1(9)$ & 10 & 1 \\
\hline CC376 & $\begin{array}{l}\text { t359 (3), t376 (1), t1198 (1), } \\
\text { t1897 (2) }\end{array}$ & 7 & 1 & 2 & & & $5(71.4)$ & & & 7 & 0 \\
\hline $\mathrm{CC} 078$ & $t 056(4), t 078(1), \mathrm{t} 258(1)$ & 6 & & 2 & & & $1(16.7)$ & & & 4 & 2 \\
\hline $\mathrm{CC} 005$ & $\begin{array}{l}\text { t005 (1), t223 (1), t712 (1), } \\
\text { t790 (1), t902 (1), t } 2439(1)\end{array}$ & 6 & & & & & $3(50)$ & $1(16.7)$ & & 4 & 2 \\
\hline $\mathrm{CC} 024$ & $t 008(2), t 024(1), \mathrm{t} 190$ (1) & 4 & & & & & $1(25)$ & & & 4 & 0 \\
\hline CC888 & $\begin{array}{l}\text { t127 (1), t160 (1), t888 (1), } \\
\text { t2433 (1) }\end{array}$ & 4 & & & & & $2(50)$ & & & 2 & 2 \\
\hline $\mathrm{CC} 230$ & $\mathrm{t} 230(1), \mathrm{t} 550(1), \mathrm{t} 571$ & 3 & & & 1 & & $3(100)$ & $1(33.3)$ & & 1 & 2 \\
\hline Others $^{a}$ & $\begin{array}{l}\text { t015 (6), t026 (4), t136 (1), } \\
\text { t153 (1), t159 (1), t163 (1), } \\
\text { t216 (1), t330 (1), t350 (1), } \\
\text { t645 (1), t777 (1), t1662 (1), } \\
\text { t1992 (1), t2435 (1), t2438 (1), } \\
\text { t2441 (1), t2442 (1), t2443 (1), } \\
\text { t2476 (1), }\end{array}$ & 28 & & 1 & 3 & 1 & $21(75)$ & & & 22 & 6 \\
\hline Total & & 101 & 2 & 5 & 17 & 2 & 70 & 5 & 2 & 80 & 21 \\
\hline
\end{tabular}

Previously published MSSA spa types are in italics [3, 8]. Empty cells denote a negative result

eta/etb exfoliative toxin $\mathrm{A} / \mathrm{B}$, etd exfoliative toxin $\mathrm{D}, P V L$ Panton-Valentine leukocidin toxin, $R$ resistant, $t s t$ toxic shock syndrome toxin

${ }^{a}$ Includes strains that were not typeable, singleton, no founder or excluded from analysis (shorter than five repeats)

b Resistant to at least one of these antibiotic compounds: levofloxacin, ciprofloxacin, norfloxacin 
[t084 (8 strains), t002 (7), t015 (6), t026 (4), t056 (4), t012 (3), t359 (3), t008 (2), t021 (2), t062 (2)] accounted for $40.6 \%$ of all isolates. Comparing our MSSA spa types with those deposited in the spa server for MRSA and MSSA strains, t002 is the fourth most frequent spa type worldwide among MSSA and MRSA isolates. t008, t015, t024, and t084 correspond to the top ten spa types in this large database (http://www.spaserver.ridom.de). Some of our spa types are similar to those previously reported in MSSA strains (Table 1, spa types in italics) [3, 8]. Genetic diversity of our study isolates exceeded that of a previously published study where 14 different spa types among 84 MSSA strains were detected [3]. A recent study from France on MSSA bloodstream infections using pulsed-field gel electrophoresis (PFGE) for genetic analysis showed a small number of PFGE divisions among isolates and were associated with epidemic phenomena in healthcare institutions [9]. If spa typing or spa-CC data were analyzed by the type of ward, no cluster with the same MSSA spa type at the same time was observed. The findings of molecular typing were supported by epidemiological data from surveillance: there was no evidence for an outbreak situation during the study period.

$S$. aureus produces a variety of extracellular toxins. They are associated with abscesses, furunculosis, and necroticing pneumonia or toxic shock syndrome $[5,6]$. The $t s t$ gene was detected in $17 \%$ of cases, etd in 5\%, PVL and etaletb each in 2\%. One isolate carried both PVL and etd (spa type t1198). Every etd-positive strain was associated with a different spa type, 17 tst-positive strains with 13 different types (4 strains of spa t002). In our study, the percentage of tst gene-positive invasive MSSA strains was comparable with MSSA strains from bloodstream infections [9] but lower than in a collection of MSSA strains mainly from sites other than blood [3]. All toxin positive strains were isolated from blood cultures except for two tst-positive strains (from soft tissue and a joint biopsy).

In contrast to recent studies from France and Germany, the antibiotic resistance rates (Table 1) of quinolones and macrolides were low [3, 9]. However, $93 \%$ and $89 \%$ of spaCC012 and spa-CC084/CC085 were penicillin-resistant, respectively. Only $5 \%$ of all strains were macrolide resistant, not allowing meaningful interpretation. The prevalence of methicillin-resistant $S$. aureus (MRSA) in our institution is low [10]; only two invasive isolates of $S$. aureus were found to be methicillin resistant and were therefore excluded.

Nine of 65 spa types representing 26.7\% of MSSA strains were also represented in our local MRSA spa database (t002, t008, t012, t015, t024, t026, t163, t230, t548). This genetic similarity between MRSA and MSSA is consistent with other reports $[3,8,11]$. However, these $s p a$ types are also frequent types worldwide and therefore importation of MRSA strains seems more likely than acquisition of methicillin-resistance genes among the local MSSA strain pool as suggested previously $[8,11]$. But the common genetic background among MSSA and MRSA strains indicates a certain selective advantage regardless of resistance and accessory virulence gene profile.

In conclusion, spa typing revealed a great genetic diversity among invasive MSSA strains consecutively isolated from primarily sterile sites without a predominant spa type or spa-CC. Besides worldwide common spa types and spa-CC, many rare and newly recognized spa types were observed. There was no evidence for MSSA crossinfections among patients. tst was the most frequent virulence gene detected among MSSA isolates. Further spa typing data on MSSA strains from other places would allow a more comprehensive understanding of the genetic background.

Acknowledgements This study was supported by the scientific research fund of the University Hospital Basel.

\section{References}

1. Kaech C, Elzi L, Sendi P, Frei R, Laifer G, Bassetti S, Fluckiger U (2006) Course and outcome of Staphylococcus aureus bacteraemia: a retrospective analysis of 308 episodes in a Swiss tertiarycare centre. Clin Microbiol Infect 12:345-352

2. Miller LG, Perdreau-Remington F, Bayer AS, Diep B, Tan N, Bharadwa K, Tsui J, Perlroth J, Shay A, Tagudar G, Ibebuogu U, Spellberg B (2007) Clinical and epidemiologic characteristics cannot distinguish community-associated methicillin-resistant Staphylococcus aureus infection from methicillin-susceptible $S$. aureus infection: a prospective investigation. Clin Infect Dis 44:471-482

3. Layer F, Ghebremedhin B, Konig W, Konig B (2006) Heterogeneity of methicillin-susceptible Staphylococcus aureus strains at a German University Hospital implicates the circulating-strain pool as a potential source of emerging methicillin-resistant $S$. aureus clones. J Clin Microbiol 44:2179-2185

4. Strommenger B, Kettlitz C, Weniger T, Harmsen D, Friedrich AW, Witte W (2006) Assignment of Staphylococcus isolates to groups by spa typing, SmaI macrorestriction analysis, and multilocus sequence typing. J Clin Microbiol 44:2533-2540

5. Lina G, Piemont Y, Godail-Gamot F, Bes M, Peter MO, Gauduchon V, Vandenesch F, Etienne J (1999) Involvement of Panton-Valentine leukocidin-producing Staphylococcus aureus in primary skin infections and pneumonia. Clin Infect Dis 29:11281132

6. Yamasaki O, Tristan A, Yamaguchi T, Sugai M, Lina G, Bes M, Vandenesch F, Etienne J (2006) Distribution of the exfoliative toxin D gene in clinical Staphylococcus aureus isolates in France. Clin Microbiol Infect 12:585-588

7. Mehrotra M, Wang G, Johnson WM (2000) Multiplex PCR for detection of genes for Staphylococcus aureus enterotoxins, 
exfoliative toxins, toxic shock syndrome toxin 1 , and methicillin resistance. J Clin Microbiol 38:1032-1035

8. Hallin M, Denis O, Deplano A, De Mendonca R, De Ryck R, Rottiers S, Struelens MJ (2007) Genetic relatedness between methicillin-susceptible and methicillin-resistant Staphylococcus aureus: results of a national survey. J Antimicrob Chemother 59:465-472

9. van der Mee-Marquet N, Epinette C, Loyau J, Arnault L, Domelier AS, Losfelt B, Girard N, Quentin R (2007) Staphylo- coccus aureus strains isolated from bloodstream infections changed significantly in 2006. J Clin Microbiol 45:851-857

10. Mertz D, Frei R, Jaussi B, Tietz A, Stebler C, Fluckiger U, Widmer AF (2007) Throat swabs are necessary to reliably detect carriers of Staphylococcus aureus. Clin Infect Dis 45:475-477

11. Aires de Sousa M, Conceicao T, Simas C, de Lencastre H (2005) Comparison of genetic backgrounds of methicillin-resistant and susceptible Staphylococcus aureus isolates from Portuguese hospitals and the community. J Clin Microbiol 43:5150-5157 